\title{
REVIEW
}

\section{What's in a Name? Redefining Type 2 Diabetes Remission}

Sanjay Kalra $\cdot$ Arbinder Singal $\cdot$ Tejal Lathia

Received: November 24, 2020 / Accepted: December 16, 2020 / Published online: January 24, 2021

(C) The Author(s) 2021

\section{ABSTRACT}

Diabetes remission is a concept that is marked by ample attention, but lack of unanimity. This opinion piece explores existing definitions of diabetes remission and proposes a contemporary and comprehensive framework to help define this clinical state. Type 2 diabetes remission is defined as a healthy clinical state characterized by achievement of HBA1c below the targeted level, maintained for at least 6 months, with or without continued use of lifestyle modification and/or metformin, provided that this is not due to complications, comorbid conditions or concomitant therapy.

S. Kalra $(\bowtie)$

Department of Endocrinology, Bharti Hospital, Karnal, India

e-mail: brideknl@gmail.com

A. Singal

Department of Pediatric Urology, Mitr Hospital, Mumbai, India

T. Lathia

Department of Endocrinology, Apollo Hospitals and

Fortis Hospital, Mumbai, India 


\section{Diabetes Remission: Infographic}

\section{DiabetesTherapy 7 OPEN}

What's in a Name? Redefining Type 2 Diabetes Remission

Kalra, S., Singal, A., Lathia, T. What's in a Name? Redefining Type 2 Diabetes Remission. Diabetes Ther (2021).
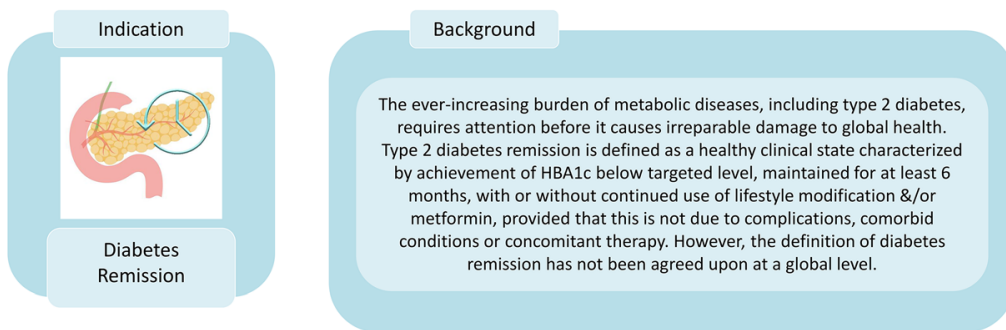

Aim

This article simplifies the current knowledge regarding diabetes remission, and suggests a pragmatic approach to counsel patients and public.

Lack of consensus in the definition suggests that these may be lack of clarity in ongoing discourse on diabetes on diabetes remission.

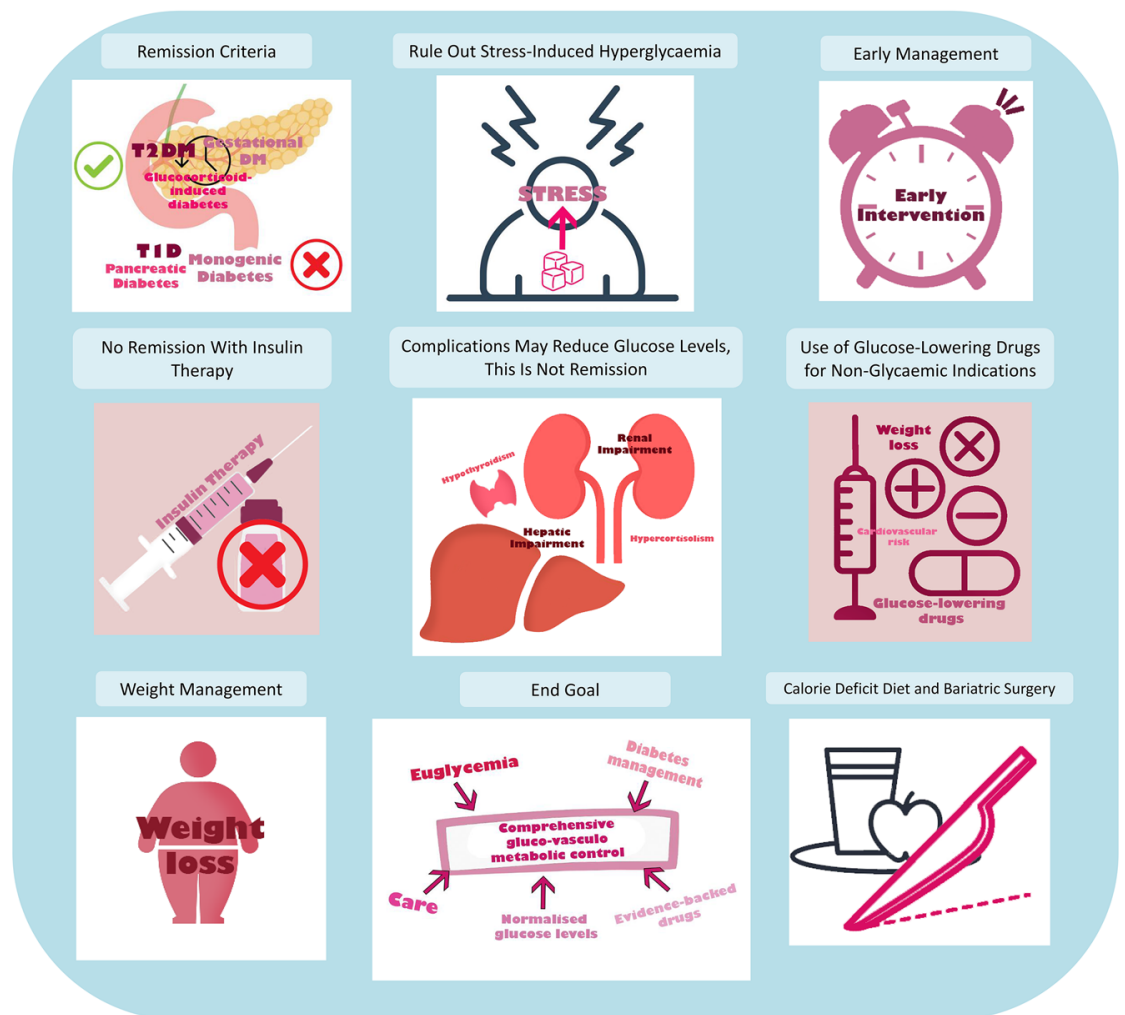


Keywords: Calorie restriction mimicry; Cure; Euglycemia; Lifestyle modification; Metformin; Remission of diabetes; Reversal of diabetes; Type 2 diabetes

\section{Key Summary Points}

Diabetes remission is promoted as an ideal endpoint of therapy.

The definition of diabetes remission is open to discussion and debate.

Type 2 diabetes remission is defined as a healthy clinical state characterized by achievement of HBA1c below the targeted level, maintained for at least 6 months, with or without continued use of lifestyle modification and/or metformin, provided that this is not due to complications, comorbid conditions or concomitant therapy.

\section{DIGITAL FEATURES}

This article is published with digital features, including a summary slide and infographic, to facilitate understanding of the article. To view digital features for this article, go to https://doi. org/10.6084/m9.figshare.13386992.

\section{INTRODUCTION}

Diabetes remission is an often-discussed topic in contemporary endocrinology. A recent position statement suggests that the remission should be the primary clinical goal of diabetes management [1]. Whether this is possible or not for persons living with established type 2 diabetes, complicated type 2 diabetes, type 1 diabetes or pancreatic diabetes is a debate in itself. In this opinion piece, we discuss evolving thoughts about the definition of diabetes remission and propose a contemporary definition for this clinical situation. This article is based on previously conducted studies and does not contain any new studies with human participants or animals performed by any of the authors.

\section{Choice of Words}

The terms 'cure,' 'remission' and 'reversal' all have been used in the context of diabetes management, either as a target or as a phase in the natural history of disease. Current consensus supports the use of the term 'remission' and agrees that it should be limited to the context of type 2 diabetes [2]. Characteristics that favor remission, such as successful weight loss, short duration of diabetes and absence of complications, have also been identified [3]. The terms 'partial remission' or honeymoon are preferred for type 1 diabetes [4].

\section{Evolution of Understanding}

However, the classic definition of remission, proposed in 2009 [5], and endorsed in 2020 [1], needs to be revisited. Enhanced interest in this field, improved understanding of glycemic targets and the need for comprehensive baro-vasculo-metabolic control, development of newer glucose-lowering drugs that can be used for non-glucose lowering indications and semantic evolution in disciplines such as oncology support the need for this discussion.

Targets for diabetes management have evolved from pure glucocentric numbers to comprehensive metabolic parameters [6]. The need for other glycemic parameters, apart from glycated hemoglobin (HbA1c), is well understood now. Similarly, targets or definitions of diabetes relapse should include the concept of metabolic well-being as well. Apart from efficacy, diabetes treatment also focuses on safety and tolerability. An ideal definition of relapse, therefore, should mention these aspects, too.

\section{The 2009 Definition}

Buse et al. (2009) [5] published a consensus statement that covered both type 1 and 2 diabetes. Remission was defined as achievement of glycemia below the diabetes range, in the 
absence of active pharmacologic or surgical therapy for at least 1 year. They further stratified remission as partial and complete, based upon the degree of glycemia achieved. Partial remission implied achievement of glycemic levels below the diagnostic thresholds for diabetes, i.e., in the prediabetic range, while complete remission meant maintenance of normal glycemic parameters for at least 1 year. The authors also created the term 'prolonged remission' to describe complete remission of at least 5 years' duration [5].

Buse et al. must be commended for their path-breaking efforts to conceptualize and define the cure of diabetes. As they have hoped, their article has engendered active discussion in the field. There are a few limitations to their proposal, however. Their definition included type 1 diabetes, which has a different pathophysiology from type 2 diabetes. It did not specify whether their framework is applicable to pancreatic diabetes or other types of diabetes. Glycemia was not defined by HbA1c alone, and the interpretation was left to the reader.

Biochemical remission may not always be synonymous with clinical well-being. An unsupervised quest for "low glucose values" may lead to eating disorders, including the newly coined orthorexia nervosa [7], and precipitate malnutrition. A definition of remission should clearly discourage overenthusiastic efforts at lowering glucose through restricted diets, which may lead to ill health. Reduction, or stoppage, of glucose-lowering therapy is seen in conditions such as chronic kidney disease. Would development of this unwelcome complication be included as a cure of diabetes?

The choice of duration (1 year and 5 years) for defining remission may be considered arbitrary. Considering that diabetes a chronic syndrome, the 1-year cutoff for diagnosis of remission can be considered reasonable. Prolonged remission, however, should not be treated as a dichotomous end point; rather persons may "be in remission for a particular number of months or years."

It is assumed that all persons labeled as being in remission would continue to carry out lifestyle modification (LSM). The definition of remission is based solely upon the glycemic burden and not upon the intensity of therapy. In 2009, the firstline therapy for diabetes was LSM. Continued adherence to or persistence with LSM was not considered relevant to the definition of remission. Today, LSM and metformin monotherapy together are considered the first-line treatment for type 2 diabetes. Metformin can also be used in prediabetes. Does it make sense, therefore, to define persons who have normal glycemic parameters or those who do not meet the diagnostic criteria for diabetes while taking metformin as being in remission? It would also be necessary to rule out a situation where stress-related hyperglycemia is misdiagnosed as diabetes and its reversal termed as remission of diabetes.

\section{The 2019 Definition}

In 2019, Nagi et al., representing the Association of British Clinical Diabetologists (ABCD) and the Primary Care Diabetes Society (PCDS), published a simpler definition of remission: the achievement of glycemia below the threshold currently used for diagnosis of type 2 diabetes and sustained for a minimum period of 6 months, with the patient having discontinued all glucose-lowering therapies [2]. A similar model had been suggested earlier by Ramos-Levi et al., who had proposed using the American Diabetes Association diagnostic thresholds for glycated hemoglobin to define remission after bariatric surgery [8].

Nagi et al.'s definition is easier to follow and does help blunt the social stigma of a 'diabetes' label, which improves patient motivation, potentially reduces insurance costs and minimizes the burden on the health care system.

The duration of remission has been specified in both definitions. As diabetes is a chronic disorder, and as glycemic levels are prone to fluctuations for various reasons, it stands to reason that a minimum duration of time must be specified. Six months of control, as mentioned in the 2019 definition, seems a fair time frame to state that a person with type 2 diabetes is in remission. Hence, prolonged remission is not included in the British rubric.

A few limitations are noted in this definition as well. Diabetes is a complex disorder, with 
multiple methods of diagnosis: fasting glucose, post-load glucose, glycated hemoglobin, random glucose and symptoms. If the diagnosis of diabetes is multifaceted, how can the definition of relapse be so simple? The target $\mathrm{HbA1c}$ is defined as being "below the threshold currently used for diagnosis of type 2 diabetes." This may seem too rigid, considering that HbA1c targets in diabetes are pegged at $7.0 \%$, with the added flexibility of modifying them based on individual needs. The need to consider other glycemic parameters, as well as markers of diabetes pathophysiology (such as insulin resistance) is not addressed.

Whether lifestyle modification or medical nutrition therapy is considered a "glucose-lowering therapy" is not clarified in the 2019 definition. The role of renal, hepatic, thyroid, adrenal or pituitary impairment in lowering glucose levels is not mentioned either. The limitation of stress-related hyperglycemia being labeled as diabetes may occur here as well.

\section{Levels of Remission}

Buse et al. used an analogy from oncology, likening hyperglycemia to tumor load [5]. The science of oncology has evolved dramatically over the past decade. Oncologists now speak in terms of cytogenic, molecular, hematologic and clinical remission in hematologic malignancies such as chronic myeloid leukemia (CML) [9]. The term "treatment-free remission" is also used when tyrosine kinase inhibitor therapy is stopped. In solid tumors such as ovarian cancer, oncologists speak of maintenance therapy after up-front treatment of cancer and of a "drugsensitive recurrence" (platinum-sensitive recurrence) [10]. These developments can be extrapolated to the diabetes care ecosystem to create contemporary definitions of remission.

\section{Diabetes Remission May Be Defined at the Metabolic, Biochemical, Clinical and Pharmacologic Levels}

To ensure clarity of thought, metabolic remission may be defined as reversal of insulin resistance or a secretory defect; (complete) biochemical remission as achievement of HbA1c below the diagnostic threshold of diabetes or clinically significant (partial) remission as achievement of HbA1c below the target set for a particular individual's management; and clinical or pharmacologic remission as the absence of need for drug therapy.

Using such a rubric creates a hierarchal system where one can progressively try to prevent pathology, and improve physiology, in a person with type 2 diabetes. All these definitions should be accompanied by the caveat that remission should not be due to concomitant complications, comorbid conditions, or medical or surgical interventions that can cause hypoglycemia. Some examples of such 'exclusion criteria' include renal or hepatic impairment, reduced appetite due to sepsis or tumors, chronic use of anti-malarial drugs/antibiotics and endoscopic devices that cause reduced food intake.

\section{Drug-Sensitive Remission}

The concept of pharmacologically sensitive remission is a challenging one, which may spark more confusion than consensus. Multiple classes of drugs are used for the management of type 2 diabetes. Some drugs such as metformin and pioglitazone are insulin sensitizers, which can be likened to the maintenance therapy of oncology, or bacteriostatic drugs used in infectious disease control. Other classes such as GLP1-RA (glucagon-like 1 peptide receptor agonists), SGLT2i (sodium glucose cotransporter-2 inhibitors) and AGIs (alpha glucosidase inhibitors) act as calorie restriction mimetics [11], with a mechanism of action that is similar to and overlaps that of bariatric surgery. Would it make sense to use the phrase 'drug-sensitive remission' or partial remission' for persons who are able to demonstrate and maintain adequate improvement in glycemic parameters while on lifestyle modification and metformin monotherapy? These phrases may also then be used for persons on GLP1RA or SGLT2i monotherapy.

This discussion becomes even more pertinent, considering the fact that these glucose- 
lowering therapies are being exapted for use in non-glycemic indications such as obesity, heart failure and chronic kidney disease [12-14]. If calorie restriction can be accepted as a part of lifestyle medicine [1], while defining diabetes remission, the use of a calorie restriction mimetic should be accepted as well.

\section{Complete vs. Partial Remission}

Taking the concept of partial and complete remission further, these terms may be used to describe partial or complete discontinuation of drug therapy. An individual with type 2 diabetes, who was previously managed with three oral glucose-lowering drugs, but is now in good control with two or one drug, may be termed as being in partial remission. This label may be used for persons who are on metformin or a caloric restriction mimetic. On the other hand, an individual who is able to maintain euglycemia without drugs can be defined as being in complete remission.

\section{The Future Is Not Far}

As we craft a comprehensive definition, we appreciate that endocrinology is a rapidly evolving branch of medicine. The way in which we measure glycemia, treat diabetes and assess glycemic targets is changing. The uses of continuous glucose monitoring systems, bariatric surgery and time in range are examples of this. It is possible, therefore, that the construct of diabetes remission may change with time. This should not detract us, however, from creating a definition that meets contemporary conditions and concerns.

\section{Contemporary Definition}

So far, our discussion has followed a deconstructive approach, focusing on meronymous aspects of diabetes remission. For clinical and research purposes, however, a holonymous definition is more appropriate.

Therefore, we propose the following definition of diabetes remission: Type 2 diabetes remission is defined as a healthy clinical state characterized by achievement of HBA1c $<7.0 \%$, maintained for at least 6 months, with or without continued use of lifestyle modification and/or metformin, provided that this is not due to complications, comorbid conditions or concomitant therapy.

Future discussants may support a more comprehensive definition, if preventive pharmacotherapy becomes the norm: Type 2 diabetes remission is defined as a clinical state of health, characterized by achievement of glycemic levels below the targeted level (as decided after shared decision making), maintained for at least 6 months, with or without continued use of lifestyle modification and/or metformin and/ or therapy that is also approved for use in nondiabetic individuals, provided that this is not due to concomitant complications, comorbid conditions or therapy that may lead to lowering of drug requirements and is not associated with untoward adverse events.

\section{Summary}

Diabetes management continues to evolve. The last few decades especially have changed our praxis related to the prevention and management of diabetes and its complications. Hopes regarding remission of diabetes have been raised. Portraying "cure of diabetes" as a goal for all persons with diabetes, irrespective of its etiology or complication status, however, is misleading and has the potential to do harm.

In such a situation, we all bear the responsibility to define and describe diabetes remission in an apt and appropriate manner. The term "remission of type 2 diabetes" should be used responsibly and rationally. This opinion piece is a step forward in this direction. We echo Buse et al. (2009) in hoping that the definition we propose will stimulate discussion and debate, lead to consensus and clarity, and finally contributes to better health for all individuals living with diabetes. 


\section{ACKNOWLEDGEMENTS}

We acknowledge the contribution of Prashant Mehta, whose expertise in medical oncology helped us frame our thoughts. We acknowledge the ideas shared by Ganapathi Bantwal and Mangesh Tiwaskar, which continue to help us enhance our understanding of the concept.

Funding. No funding or sponsorship was received for this study or publication of this article. No editorial assistance, including medical writing assistance, has been used in preparation of the manuscript.

Authorship. All named authors meet the International Committee of Medical Journal Editors (ICMJE) criteria for authorship for this article, take responsibility for the integrity of the work as a whole, and have given their approval for this version to be published.

Disclosures. SK and TL have received advisory fees from and AS is CEO and Founder of Fitterfly Technologies Pvt Ltd. SK is also on the Editorial Board of Diabetes Therapy.

Compliance with Ethics Guidelines. This article is based on previously conducted studies and does not contain any new studies with human participants or animals performed by any of the authors.

Data Availability. Sharing is not applicable to this article as no datasets were generated or analyzed during the current study.

Open Access. This article is licensed under a Creative Commons Attribution-NonCommercial 4.0 International License, which permits any non-commercial use, sharing, adaptation, distribution and reproduction in any medium or format, as long as you give appropriate credit to the original author(s) and the source, provide a link to the Creative Commons licence, and indicate if changes were made. The images or other third party material in this article are included in the article's Creative Commons licence, unless indicated otherwise in a credit line to the material. If material is not included in the article's Creative Commons licence and your intended use is not permitted by statutory regulation or exceeds the permitted use, you will need to obtain permission directly from the copyright holder. To view a copy of this licence, visit http://creativecommons.org/licenses/by$\mathrm{nc} / 4.0 /$.

\section{REFERENCES}

1. Kelly J, Karlsen M, Steinke G. Type 2 Diabetes Remission and Lifestyle Medicine: A Position Statement from the American College of Lifestyle Medicine. Am J Lifestyle Med. 2020;14(4):406-19.

2. Nagi D, Hambling C, Taylor R. Remission of type 2 diabetes: a position statement from the Association of British Clinical Diabetologists (ABCD) and the Primary Care Diabetes Society (PCDS). British J Diabetes. 2019;19(1):73-6.

3. Hopkins MD, Taylor R, Lean ME. The DiRECT principles: giving Type 2 diabetes remission programmes the best chance of success. Diabet Med. 2019;36(12):1703-4.

4. Zhong T, Tang R, Gong S, Li J, Li X, Zhou Z. The remission phase in type 1 diabetes: Changing epidemiology, definitions, and emerging immunometabolic mechanisms. Diabetes Metabol Res Rev. 2020;36(2):e3207.

5. Buse JB, Caprio S, Cefalu WT, Ceriello A, Del Prato $\mathrm{S}$, Inzucchi SE, et al. How do we define cure of diabetes? Diabetes Care. 2009;32(11):2133-5.

6. Kalra S, Baruah MP, Sahay R, Kishor K. Pentads and hexads in diabetes care: numbers as targets; numbers as tools. Indian J Endocrinol Metabol. 2017;21(6):794-6.

7. Ramos-Levi AM, Cabrerizo L, Matía P, SánchezPernaute A, Torres AJ, Rubio MA. Which criteria should be used to define type 2 diabetes remission after bariatric surgery? BMC Surg. 2013;13(1):8.

8. Dunn TM, Bratman S. On orthorexia nervosa: A review of the literature and proposed diagnostic criteria. Eat Behav. 2016;21:11-7.

9. Hochhaus A, Baccarani M, Silver RT, Schiffer C, Apperley JF, Cervantes F, et al. European LeukemiaNet 2020 recommendations for treating chronic myeloid leukemia. Leukemia. 2020;34:966-84. 
10. Walsh CS. Latest clinical evidence of maintenance therapy in ovarian cancer. Curr Opin Obstet Gynecol. 2020;32(1):15-21.

11. Kalra S, Jacob JJ, Gupta Y. Newer antidiabetic drugs and calorie restriction mimicry. Indian J Endocrinol Metabol. 2016;20(1):142-6.

12. Mancini MC, de Melo ME. The burden of obesity in the current world and the new treatments available: focus on liraglutide $3.0 \mathrm{mg}$. Diabeto Metabol Syndrome. 2017;9(1):44. https://doi.org/10.1186/ s13098-017-0242-0.
13. McMurray JJ, Solomon SD, Inzucchi SE, Køber L, Kosiborod MN, Martinez FA, et al. Dapagliflozin in patients with heart failure and reduced ejection fraction. N Engl J Med. 2019;381(21):1995-2008.

14. Heerspink HJ, Stefánsson BV, Correa-Rotter R, Chertow GM, Greene T, Hou FF, et al. Dapagliflozin in patients with chronic kidney disease. N Engl J Med. 2020;383:1436-46. 